Network Working Group

Request for Comments: 300

25 January 1972

Obsoletes: RFC 211 NIC 7191

NIC 8468

\title{
ARPA NETWORK MAILING LISTS
}

The following are three lists to be used for distribution of Network documents. List A should be used by sites when they distribute their own documents, to get RFC's to Liaisons as quickly as possible. All but local mail should be sent Air Mail. Lists B and C will be used by NIC in making distribution of copies to non-site Network participants and to Station Agents.

For phone numbers and lists of names by sites see the Current Directory of Network Participants.

\section{A. INITIAL DISTRIBUTION LIST}

Note: This list includes all Network Liaisons and others who should receive initial distribution of $\mathrm{RFC}^{\prime} \mathrm{s}$, whether sent from NIC or from sites. They will also receive selected catalogs and other formal documents from NIC.

$\mathrm{AMES}-\mathrm{CD}$

A. Wayne Hathaway

Mail Stop 233-9

NASA Ames Research Center

Moffett Field, Calif. 94035

AMES-ILLIAC

John W. McConnell

NASA Ames research Center

Mail Stop 202-10

Moffett Field, Calif. 94035

ARPA

Steve D. Crocker

Advanced Research Project Agency

1400 Wilson Boulevard

Arlington, Virginia 22209
CMU

Harold R. Van Zoeren

Carnegie-Mellon University

Computer Science Department

Schenley Park

Pittsburgh, Pa. 15213

DOCB

Schuyler Stevenson R-523

Department of Commerce NOAA

325 South Broadway

Boulder, Colorado 80302

HARV

Robert L. Sundberg

Harvard University

Aiken Computation Laboratory

33 Oxford Street

Cambridge, Mass. 02138 


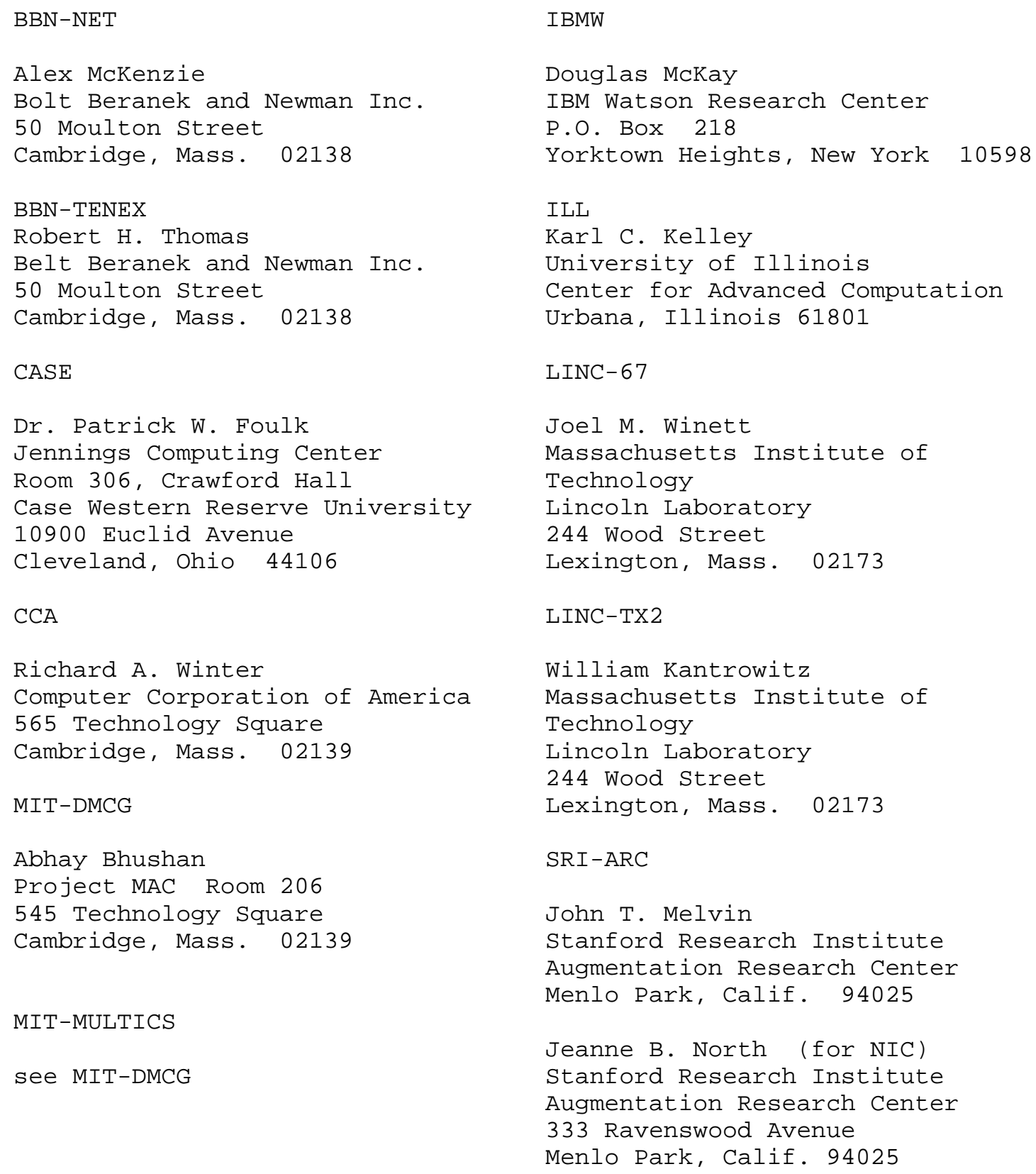


MITRE

Peggy M. Karp

MITRE Corporation

Information Systems Dept., W140

Westgate Research Park

McLean, VA 22101

NBS

Thomas N. Pyke, Jr.

National Bureau of Standards

Center for Computer Sciences and

Technology

Washington, D.C. 20234

NIC

see SRI-ARC

RADC

Thomas S. Lawrence

Rome Air Development Center (ISIM)

Griffiss Air Force Base

Rome, New York 13440

RAND

John F. Heafner

Rand Corporation

Computer Science Department

1700 Main Street

Santa Monica, Calif. 90406

RAY

Thomas O'Sullivan

Raytheon Data Systems

1415 Boston-Providence Turnpike

Norwood, Mass. 02062
SU-AI

James A. (Andy) Moorer

Stanford University

Computation Center, AI Project

Stanford, Calif. 94305

SU-HP

Edward A. Feigenbaum

Stanford University

Heuristic Programming

Serra House

Stanford, Calif. 94305

$\mathrm{UCLA}-\mathrm{CCN}$

Robert T. Braden

University of California at

Los Angeles

5308 Math Sciences Building

Los Angeles, Calif. 90024

$\mathrm{UCLA}-\mathrm{NMC}$

Ari O. Ollikainen

University of California at

Los Angeles

Computer Science Department

3732 Boelter Hall

Los Angeles, Calif. 90024

UCSB

James E. White

University of California at

Santa Barbara

Computer Research Laboratory

Santa Barbara, Calif. 90024 
SDC

Abe S. Landsberg

System Development Corporation 2500 Colorado Avenue

Santa Monica, Calif. 90406

SRI-AI

B. Michael Wilber

Stanford Research Institute Artificial Intelligence Group 333 Ravenswood Avenue

Menlo Park, Calif. 94025

USC

James Pepin

University of Southern California

School of Engineering

Los Angeles, Calif. 90007

UTAH

Barry D. Wessler

University of Utah

Computer Science/IRL

Salt Lake City, Utah 84112
$\mathrm{UCSD}-\mathrm{CC}$

Charles Holland

Computer Center

University of California at

San Diego

La Jolla, Calif. 90037

USAF-ETAC

Capt. George N. Petregal

USAF-ETAC

Bldg. 159

Navy Yard Annex

Washington, D.C. 20333 
Note: These Network participants will receive all formal Network documents sent from NIC, in the mailing to Liaisons when such is made, otherwise at the time documents are sent to station Agents.

$\mathrm{CCCTF}$

C. D. Shepard

Canadian Computer Communications Task Force

100 Metcalfe Street

4 th Floor

Ottawa 2, Canada

DART

Prof. Robert F. Hargraves Kiewit Computation Center Dartmouth University Hanover, New Hampshire 03755

EDUCOM

John LeGates

EDUCOM

44 School Street

Boston, Mass. 02108

MERIT

E. M. Aupperle

MERIT Computer Network

University of Michigan

1037 N. University Building

Ann Arbor, Michigan 48104

$\mathrm{NETH}$

Tjaart Schipper

13 Marijkestraat

Leiderdorp, Netherlands
WATERU

Prof. Donald Cowan

Dept. of Computer Science

University of Waterloo

Waterloo, Ontario, Canada

CHIU

Prof. Robert L. Ashenhurst

Institute for Computer Research

University of Chicago

Chicago, Illinois 60637

NPL

Derek Barber

Computer Science Division

National Physical Laboratory

Teddington, Middlesex, England

$\mathrm{UBC}$

Dave Tywer

Computing Center

University of British Columbia

Vancouver 8, Canada

NSF

Dr. D. D. Aufenkamp

Office of Computing Activities

National Science Foundation

1800 G Street, N.W.

Washington, D. C. 20550 
SUNY

Prof. Art J. Bernstein

SUNY Stoneybrook

Dept. of Computer Science

Stoneybrook, L.I., N.Y. 11790

UCI

Prof. David Farber

Dept. of Information and Computer Science

University of California

Irvine, Calif. 92664

UKICS

Prof. Peter Kirstein

Institute of Computer Science

University of London

44 Gordon Square

London, W.C.1, England

WASHU

Marianne Pepper

Washington University

Computer Systems Laboratory

724 South Euclid Avenue

St. Louis, No. 63110 
Note: Station Agents receive copies from NIC of all documents distributed to Liaisons, as well as documents sent as part of a Station collection. A mailing is made to station Agents once a week, or oftener when quantity warrants.

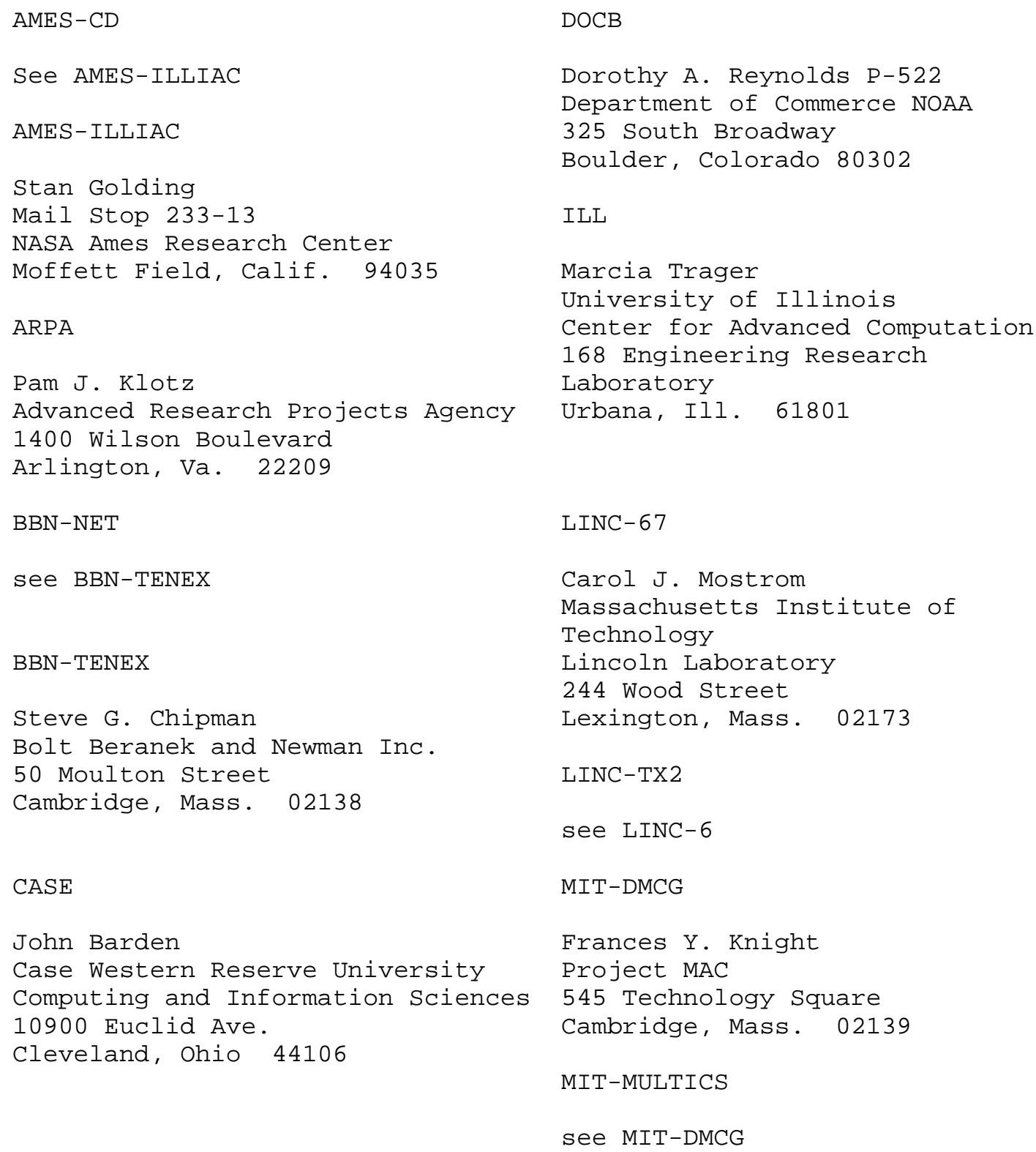


CCA

Martha A. Ginsberg

Computer Corporation of America

565 Technology Square

Cambridge, Mass. 02139

CMU

Nancy R. Teater

Carnegie-Mellon University

Computer Science Department

Schenley Park

Pittsburgh, Pa. 15213

HARV

Bradley A. Reassow

Harvard University

Aiken Computation Laboratory

33 Oxford Street

Cambridge, Mass. 02138

RADC

Marcelle D. Petell

Rome Air Development Center (ISIM)

Griffiss Air Force Base

Rome, New York 13440

RAND

Linda M. Connelly

Rand Corporation

Computer Science Department

1700 Main Street

Santa Monica, Calif. 90406

RAY

Laura White

Raytheon Data Systems

1415 Boston-Providence Turnpike

Norwood, Mass. 02062
MITRE

Ernest H. Forman

MITRE Corporation

Information Systems Dept.,

W150

Westgate Research Park

McLean, Va. 22101

NBS

Mrs. Shirley W. Watkins

National Bureau of Standards

Bldg. 225, Room B216

Washington, D.C. 20234

NIC

see SRI-ARC

UCLA- [NMC ]

Anita Coley

University of California at

Los Angeles

Computer Science Department

3732 Boelter Hall

Los Angeles, Calif. 90024

$\mathrm{UCSB}$

Connie Rosewall

University of California at

Santa Barbara

Computer Research Laboratory

Santa Barbara, Calif. 93106

UCSD-CC

Charles Hollano

Computer Center

University of California at

San Diego

La Jolla, Calif. 90037 


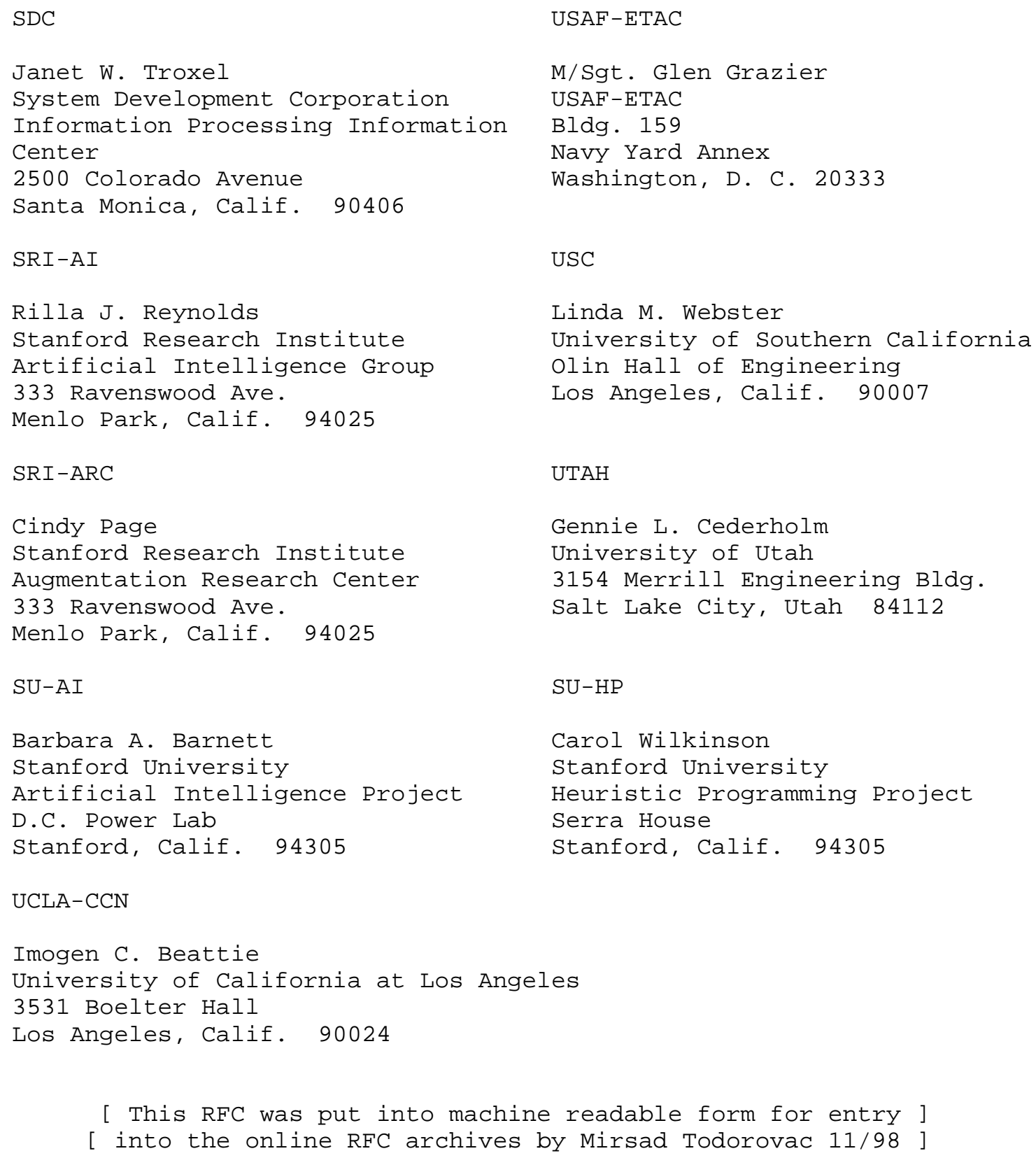

